\title{
INTERNATIONAL INQUIRY ON NEUROLOGICAL TERMINOLOGY AND PROGNOSIS IN PARAPLEGIA AND TETRAPLEGIA
}

\author{
Compiled by L. S. MichaELis, M.D. * \\ Swiss Paraplegic Centre, Basle, Switzerland
}

Results of an international inquiry, based on replies by: Abramson (New York); Bedbrook (Perth, W. Australia); Bors and Comarr (Long Beach, Cal.); Cheshire (Melbourne); Dollfus (Mulhouse); Fromm (Frankfurt/M.); Grogono (Manitoba); Grossiord (Garches); Guttmann, Walsh, Melzak, Frankel (Stoke-Mandeville); Hancock (Swansea); Hardy (Sheffield); Harris (Edinburgh); Houssa and Tricot (Brussels); Jousse (Toronto); Key and Retief (Pretoria); Klingler (Basle); Lemberg (Bayreuth); Lipschitz (Fohannesburg); Maglio (Rome); Maury (Fontainebleau); Meinecke (Bochum); Miedema and Verkuyl (T'Hoogstraat); Nakamura (Beppu, fapan); Neubauer (Tobelbad); Paeslack (Heidelberg); Rossier (Geneva); Sarrias (Barcelona); Schäfer (Zürich); Silver (Southport); Steinmann (Berne); Talbot (Boston); Wahle (Cologne); Weber (Zürich); Weiss (Warsaw); Young (Cleveland, Ohio).

DURING the first half of 1967,49 doctors working in the field of paraplegia in $4 \mathrm{I}$ departments in 16 countries received a circular inviting their collaboration which involved completion of two questionnaires. Their purpose was to establish, as far as possible, international agreement on neurologial terminology and to compare opinions on the best time for making a neurological prognosis. Observations on unusual patterns of neurological development were also asked for.

Forty-two colleagues from 35 departments in 15 countries kindly co-operated. Sixteen of them had seen more than Iooo para- and tetraplegics, seven more and 20 less then 500 patients. Twelve colleagues sent, in addition to the completed questionnaires, letters containing comment and criticism.

While all approved of the purposes of the inquiry, some felt that part of the second questionnaire was not formulated clearly enough.

\section{THE REPLIES}

(Analysed and commented on in the order of the questionnaires)

\section{NEUROLOGICAL TERMINOLOGY}

\section{The Definition of the Level of a Lesion}

(a) by the number of the most distal uninvolved segment of the cord, stating the skeletal level as well (e.g. paraplegia, complete or incomplete, below D I , due to fracture-dislocation of D9/IO vertebrae). Used by I9 authors.

(b) By the number of the most proximal involved segment of the cord, stating the skeletal level as well (e.g. paraplegia, complete or incomplete, at Di2 due to fracture-dislocation of D9/IO vertebrae). Used by 21 authors.

* Present address: c/o Stoke Mandeville Hospital, nr. Aylesbury, Bucks, England. 
(c) By the number of injured vertebrae, with a statement on neurological involvement (e.g. fracture-dislocation of $\mathrm{D} 9 / \mathrm{Io}$ vertebrae with paraplegia or paraparesis). Used by 3 authors.

Comment. Two of the colleagues who so far used method $(c)$ have since adopted method $(a)$.

Only one participant still uses method $(c)$, but it is well known that the skeletal definition of a level alone is still widely used by traumatological, orthopaedic and surgical departments-not included in this inquiry. In all countries some of these departments are still called upon to undertake the initial treatment of traumatic para- and tetraplegia. On transfer of a patient to a spinal centre their reports often lack detailed records of neurological examinations, both before and after the use of conservative, manipulative or operative methods of reduction.

Obviously such records cannot be used as evidence for or against the merits of the form of treatment employed.

One can only hope that in future a sound neurological basis for assessment will be provided everywhere so that valid conclusions can be drawn.

The two neurological methods $(a)$ and $(b)$ are at present employed by an equal number of authors.

It is relevant to recall that the two oldest and largest centres (Stoke Mandeville, 1944; Long Beach, 1946) use method (a).

The reason is not hard to find. Defining the level as below the most distal uninvolved segment is unequivocal, while there is no unanimity as to the exact meaning of a lesion at a certain level (see Paeslack and Meinecke during the preliminary discussion, 1967).

Method (a) also permits a precise definition of lesions which are-or become -incomplete in proximal segments while remaining complete distally (e.g. transverse spinal cord syndrome incomplete below $\mathrm{C}_{4}$, complete below C6). Method (a) finally goes some way towards satisfying the neuropathologist (Wolman, 1967) who pointed out that longitudinal damage both proximally and distally to the clinically defined neurological level is often found at autopsy.

Any apparent discrepancy between the clinically ascertainable detail of loss of function and structural damage found at autopsy is due to one of three causes:

I. The patient may terminally not have been fit to co-operate in the testing of muscular power and the limits of sensory loss.

2. Clinical examination may not have been thorough or recent enough or at least not recorded shortly before death.

3. In cases of late deaths histological-and at times macroscopic-changes seen at autopsy, e.g. cysts, may have developed too gradually during life to produce clinically detectable additional loss of function. (Compare the time-lag between the growth of benign tumours of the cord and the appearance of clinically discoverable signs of neurological involvement).

\section{Grading of Active Muscular Power}

For the grading of the power of partially paralysed or recovering muscles the method proposed by the British Medical Research Council (1942) ( $5=$ normal, $4=$ almost normal, $3=$ sufficient to counteract gravity, $2=$ weak, $I=$ trace or flicker only) or a very similar variant is being employed by all but four participants. Grading according to Mayo (1963) or Lovett (I912) is being used by one author 
each. The two remaining authors differentiate between slight, moderate and severe loss of power.

\section{Definition of Types of Incomplete Lesions}

In addition to detailed individual records, I4 authors use the term 'subtotal lesions' and eight use 'central syndrome', 'anterior or posterior cord syndrome' and 'epiconal lesion'.

In recovering lesions progress is charted either on special sheets of text or on successive sketches.

Comment. We doubt the usefulness of the term subtotal. It is as vague as the unqualified 'incomplete', which may mean anything between sacral sparing and slight residual paresis.

Neubauer and Lemberg emphasised that for insurance reports and correspondence with non-specialists and laymen neurological definitions alone were not enough. Classification according to independence and ability to work had to be added. In more than one centre (Cheshire, Meinecke) such classification is being worked out.

\section{NEUROLOGICAL PROGNOSIS}

The second questionnaire asked for an expression of opinion as to the right time for making a firm prognosis of the chances of neurological recovery in traumatic para- and tetraplegia in:

\section{Initially Complete Lesions}

Within 24 hours of the Accident. A firm prognosis possible in tetraplegics (8 authors); in paraplegics (10 authors); in c. equina 1. (3 authors).

Within three weeks of the Accident. Firm prognosis possible in tetraplegics (IO authors); in paraplegics (I2 authors); in c. equina 1. (3 authors).

Within six weeks of the Accident. Firm prognosis possible in tetraplegics (I5 authors); in paraplegics (I0 authors); in c. equina 1. (23 authors).

Longer periods for all levels were considered necessary by several authors.

Comment. That relatively many authors should be prepared to make a confident neurological prognosis, i.e. an estimate whether the lesion was going to remain complete, as early as 24 hours after the accident, is rather surprising. Do not spinal shock and oedema very often blur the picture during the first few weeks ? This has certainly been my own experience and I feel that in paraplegics three weeks, in tetraplegics six weeks are required, before one can reasonably exclude the possibility of partial recovery of potential value to the patient.

There is one exception to this rule. If patients are able to remember the first few minutes following the accident and report that they lost all sensation and movement at once, then the chances of recovery are very slight indeed. If paralysis takes minutes or hours to become complete at the first examination, they are slightly better. Unfortunately most patients are dazed or even unconscious for a short period and unable to provide this information.

\section{Initially Incomplete Lesions}

Opinions were invited on the right time for a prognosis as to the likely extent of further recovery in patients with incomplete lesions from the time of the arcident. 
(I) those who had only sacral sparing;

(2) those with complete motor-paralysis but 'substantial' sensory sparing;

(3) those with incomplete motor and sensory paralysis from the start.

In all these cases the majority of authors were in favour of postponing judgement for 6 months.

One author would not give an opinion until two years after the accident.

Comment. To answer these questions was considered difficult or impossible by several colleagues (Grossiord, Klingler, Maury, Meinecke, Silver, Wahle). A sharper definition of 'substantial' sensory sparing was demanded by Cheshire. Together with Grossiord and Hardy he also pointed out how frequently motor and sensory recovery are dissociated. Recovery of posterior column sensation alone was also emphasised.

This criticism of the way the questions were formulated is justified. In trying to be concise the writer failed to go into sufficient detail.

\section{UNCOMMON CHANGES OF LEVEL AND TYPE OF PARALYSIS}

In this final section of the second questionnaire an attempt was made to establish how common certain patterns of neurological pro- and regression were, and whether there were significant differences of experience between the various countries and centres.

\section{New Lesions}

$A$ rise in level by more than three segments within a few hours of the accident was occasionally seen by many authors who admitted new lesions. In one centre they amounted to a quarter of all new lesions, elsewhere percentages between I and Io per cent. were given. A rise of one or two segments is, of course, common.

The same authors had also seen a fall of level by more than three segments, predominantly sensory, within the first few weeks. Lipschitz puts their number as high as 60 per cent. Cheshire had seen at least 30 cases. Again the majority of observations amount to I to Io per cent. Falls by one or two segments were very common.

Comment. Since in some of the patients a rapid rise of level may lead to an indication for laminectomy in order to release a subarachnoid haematoma, it seemed not unimportant to find out how often this emergency occurred.

\section{Old Lesions}

True motor recovery in a previously completely paralysed muscle more than two years after injury was seen occasionally by one-half of the authors in cauda equina lesions.

A substantial rise of neurological level several years after injury was described as rare by about one half of the authors. Frankel drew attention to the fact that, in some dorso-lumbar lesions, signs of incomplete cervical involvement may be found where the dorsal intervening segments are normal.

Severe permanent increase of spasticity several years after injury without discoverable cause was seen by about one-half of the authors, rarely but definitely. Lemberg rightly emphasised how difficult it may be to be certain of the absence of such an extramedullary cause.

The opposite, a spontaneous gradual replacement of spasticity by flaccidity several years after injury, was seen only very rarely by a few authors, once by the writer. 


\section{SUMMARY AND RECOMMENDATIONS}

I. Definition of the level of a lesion. Wherever para- and tetraplegic patients are treated, the exact neurological level ought to be established and, together with a description of the skeletal injury, used to define the lesion.

Of the two different methods of defining the neurological level method $(a)$ giving the number of the most distal uninvolved segment of the cord is unequivocal. It should, wherever possible, be adopted, particularly in centres planned in future.

All future publications should state whether they employ method $(a)$ or $(b)$.

2. Assessment of active power in individual muscles is, in the great majority of centres, carried out according to the scale of the British Medical Research Council. This method should be adopted universally in future.

3. The description of incomplete lesions, apart from recording all individual detail, relies on a number of subheadings (anterior, central and posterior cordsyndromes, Brown-Sécard).

An additional method of classification in practical terms of independence and functional ability will be internationally discussed.

4. A firm prognosis as to the chances of neurological recovery in initially complete lesions might best be postponed for three weeks in paraplegia, for six weeks in tetraplegia. In initially incomplete lesions it may be wise to wait at least six months.

5. In new lesions a rise-or fall-of level by more than three segments within hours, days or weeks of injury is seen in a, generally small, percentage of cases.

6. In old lesions genuine recovery of power in a muscle completely paralysed for two years after injury is seen occasionally in cauda equina lesions.

7. A rise of neurological level several years af ter injury, at times the occurrence of cervical signs above a free interval in dorso-lumbar lesions, is seen infrequently.

8. Severe permanent increase in spasticity several years after injury-without discoverable cause-is seen as rarely as the opposite, spontaneous gradual transformation from a spastic into a flaccid lesion.

The writer thanks his co-authors for spending some of their valuable time on replying to this inquiry and for adding to its usefulness by their criticism and comment. 